\title{
Richesse spécifique et phytomasse des sous-bois de peupleraies cultivées en bordure de Garonne (Sud-Ouest de la France)
}

\author{
Marc Laquerbe* \\ Centre d'Écologie des Systèmes Aquatiques Continentaux, UMR CNRS-UPS C 5576, 29, rue Jeanne Marvig, BP 4349, \\ 31055 Toulouse Cedex 4, France
}

(Reçu le 24 janvier 2000; accepté le 17 avril 2000)

\begin{abstract}
Résumé - Les peupleraies cultivées occupent de vastes surfaces dans la plaine alluviale de la Garonne. Des communautés végétales s'y développent en sous-bois, perturbées par des pratiques d'entretien (disquage) dont l'intensité et la fréquence varient en fonction de l'âge des peupleraies. Nous nous sommes intéressés à l'influence de ce type d'entretien sur les communautés de sous-bois. Quatre peupleraies ont été choisies afin d'illustrer les différents types d'entretien. Les prélèvements d'espèces de sous-bois ont été effectués à des dates tenant compte de la phénologie des espèces et des périodes d'entretien. À l'échelle des peupleraies, la richesse spécifique des communautés végétales de sous-bois est d'autant plus élevée que le niveau d'entretien (intensité et fréquence) est plus marqué. Cette tendance est essentiellement due à des espèces annuelles et secondairement, bisannuelles. L'analyse réalisée à partir de quadrats répartis au hasard dans les peupleraies montre que la richesse spécifique des plantes de sous-bois s'accroît en fonction de l'augmentation de l'intensité et de la fréquence de la perturbation créée par l'entretien, alors que cette relation est inversée en ce qui concerne les phytomasses. Cette double tendance est observée entre les peupleraies et au sein des quadrats d'une même peupleraie. Dans ce dernier cas, les différences observées sont moins significatives. Des interactions apparaissent entre le niveau général d'entretien d'une peupleraie et l'exposition à cet entretien de différents définies au sein de cette peupleraie. La richesse spécifique des localisations graduellement plus exposées à l'entretien augmente jusqu'à une valeur seuil, au-delà de laquelle elle diminue. Ce seuil n'est atteint que dans le cas de la peupleraie dont le niveau d'entretien est le plus élevé. Ces résultats sont discutés dans le cadre de la théorie des perturbations écologiques. Les peupleraies, souvent considérées comme des facteurs de banalisation du paysage, constituent, en fait, des milieux riches et diversifiés; elles peuvent jouer un rôle majeur dans le contrôle de la diversité des écosystèmes adjacents.
\end{abstract}

perturbation / richesse spécifique / phytomasse / sous-bois / peupleraies cultivées

\begin{abstract}
Species richness and phytomass in understory cultivated poplar groves at the margin of the Garonne (South-West France). Cultivated poplar groves occupy an important part of the Garonne alluvial lowlands. Plant communities belonging to the understory, are disturbed by upkeep (disking), i.e. weeding by a metallic disk which totally destroys and buries vegetation. This upkeep differs among poplar groves: the intensity and the frequency of which decreases with the age of the poplar grove and it totally disappears during the years preceding clearing. The four poplar groves chosen for this study showed different upkeep levels. Understory species were sampled taking into account the species phenology and upkeep periods, in order to study the dynamics of the species richness and phytomass of plant communities in the understory between March et September. At the stand scale, the species richness of plant communities of the understory increased accordingly to the upkeep level (intensity and frequency). This trend was mainly due to annual species and secondarily to biennials ones. The analyses achieved from quadrat areas located at random in the studied poplar groves showed that the plant species richness of the understory communities increased, depending on the
\end{abstract}

* Correspondance et tirés-à-part

Tél. (33) 05622699 74; Fax. (33) 05622699 99; e-mail : laquerbe @ cesac.cemes.fr 
disturbance intensity induced by the upkeep, whereas this relation was inverted for phytomass. These two distinct trends were observed in inter-poplar groves comparison as well as in intra-poplar groves comparison. Interactions were observed between the general upkeep level of a poplar grove and the exposure to this upkeep of sites within this poplar grove. The species richness of sites gradually more exposed to the upkeep increased up to a threshold value, beyond which the species richness values decreased. This threshold was reached only for the poplar grove of which the general upkeep level was the most important. The results are discussed in relation to the ecological disturbance theory. When the upkeep frequency decreases, herbaceous, rhizomeous, then woody species take place. The understory plant communities of cultivated poplar groves constitute a rich and diversified zone. Their implantation into floodplain is important for adjacent ecosystem diversity.

disturbance / species richness / phytomass / understory / cultivated poplar grove

\section{INTRODUCTION}

Deux théories principales tentent d'expliquer les variations locales de richesse spécifique des communautés. Celle de l'équilibre dynamique [17] prédit le niveau de richesse spécifique connaissant le régime de perturbation et la productivité. Celle de la compétition pour les ressources [36] prédit les niveaux de richesse spécifique connaissant l'hétérogénéité spatiale des ressources et la productivité. La première insiste sur la notion de perturbation, la seconde sur celle d'hétérogénéité spatiale. L'idée selon laquelle ces deux facteurs contrôlent, avec la productivité, la richesse spécifique des communautés végétales est largement répandue [15, 32]. Dans certains milieux, notamment artificialisés, les régimes de perturbation sont suffisamment forts pour conduire à l'idée qu'ils peuvent jouer un rôle majeur dans la dynamique de la biodiversité. Les peupleraies constituent de tels milieux, les régimes de perturbation y étant déterminés, pour une large part, par l'entretien.

Dans la plaine alluviale de la moyenne vallée de la Garonne, les peupleraies cultivées occupent des superficies relativement élevées [10]. Elles bénéficient ainsi des caractéristiques des corridors riverains. À l'interface entre la terre et l'eau, ces derniers offrent des sols à fort pouvoir nutritionnel [22] et à hétérogénéité spatiale très marquée [24]. Associée à une forte dynamique naturelle des zones riveraines, cette complexité structurale s'accompagne d'une diversité spécifique et fonctionnelle élevée [40]. Cependant, la forte implantation de peupleraies artificielles conduit à une fragmentation et une uniformisation du couvert forestier [10].

Les plantations de peupliers ont avant tout un rôle de production de bois; aussi, un entretien régulier doit-il réduire le développement de la végétation de sous-bois, afin de permettre une meilleure croissance des arbres [27]. Le peuplier est très sensible à la concurrence végétale. Ces types d'entretien, appelés disquage, résultent d'un sarclage réalisé au moyen de disques métalliques qui détruisent la végétation du sous-bois et l'enfouissent sur place. Ils définissent ainsi des régimes de perturba- tions au sens de [41] : «altérations physiques soudaines qui orientent l'évolution des communautés en place». On sait que l'intensité, la fréquence et la période à laquelle elle se produit sont des éléments essentiels d'appréciation de tout phénomène de perturbation [14]. En outre, les peupleraies présentent l'intérêt d'offrir un double gradient d'intensité et de fréquence : d'une part d'une peupleraie à l'autre, à la suite d'entretiens différents, et d'autre part, au sein d'une même peupleraie, par une exposition variable à l'entretien. Comme tout système agricole, les peupleraies présentent un sous-bois dont la nature reflète non seulement l'influence de l'habitat, où elle est plantée, mais aussi celle des pratiques culturales effectuées [2].

Les perturbations ont ainsi une forte implication sur les caractéristiques des communautés végétales. Des indices tels que la richesse spécifique [1], la biomasse [35] ou la densité [4] sont fortement corrélés à la nature des perturbations. Nous utiliserons la richesse spécifique et la phytomasse pour évaluer l'impact de l'entretien dans la structuration des communautés de sous-bois. Comme l'a remarqué Lefeuvre [23], bien trop d'études se sont contentées de décrire de façon très générale la nature de ce sous-bois, ce qui a mené bien souvent à parler des peupleraies plantées comme des facteurs de banalisation de la flore. Le but de cette étude est de (1) caractériser cette végétation de sous-bois, (2) d'étudier les conséquences de ce double gradient de perturbations, sur deux attributs, richesse spécifique et phytomasse, des communautés de sous-bois des peupleraies cultivées et enfin (3) de donner une idée sur le rôle que peut avoir ce type d'exploitation sur le milieu naturel.

\section{MATÉRIEL ET MÉTHODES}

\subsection{Zone étudiée}

L'expérimentation a été menée en 1995 dans des peupleraies situées dans la plaine alluviale de la moyenne vallée de la Garonne, à environ $40 \mathrm{~km}$ au nord de 
Toulouse dans la commune de Verdun-sur-Garonne ( $43^{\circ} 49^{\prime}$ de latitude nord, $1^{\circ} 15^{\prime}$ de longitude est), en rive gauche. Cette zone est placée à l'intérieur d'un méandre de la Garonne à 500-700 mètres des rives, hors d'atteinte des crues annuelles. Quatre peupleraies, nommées P1, $\mathrm{P} 2$, P3 et P4, font l'objet de notre étude. Chacune d'elles a été implantée à la suite de l'abattage d'une peupleraie plus ancienne. Adjacentes l'une de l'autre, leurs superficies respectives sont égales à 1,$3 ; 1,6 ; 1,6$ et 1 ha. Les arbres, espacés de $7 \mathrm{~m}$, sont des Populus x euramericana cv. I 4551. Le substrat est limono-argileux en surface et sablo-limono-argileux au-dessous de soixante $\mathrm{cm}$. Le $\mathrm{pH}$ varie de 7,4 à 8,5.

La végétation naturelle est représentée, le long du fleuve, par une saulaie-peupleraie à Salix alba et Populus gr. nigra et, au sein des terres, par une frênaieormaie à Fraxinus angustifolia subsp. oxycarpa et Ulmus minor, avec introduction massive de Robinia pseudoacacia.

\subsection{Caractéristiques de la perturbation}

La peupleraie P1 est âgée de 5 ans. Son disquage est croisé, c'est-à-dire que le disque d'entretien passe selon deux directions de part et d'autre des arbres (figure 1). Les peupliers étant jeunes, une fréquence de trois passages par an est nécessaire. La peupleraie P2 est âgée de 13 ans. L'entretien y est également croisé, mais au rythme de deux passages par an, ses peupliers adultes étant moins exigeants. La peupleraie P3 est du même âge que P2 mais son accès difficile a conduit à réaliser un disquage simple, c'est-à-dire sur une seule direction et à la fréquence d'un seul passage par an. La peupleraie P4 est âgée de 18-20 ans. Devant être abattue, elle a été laissée à l'abandon depuis 1991. Ces quatre peupleraies décrivent donc un gradient de perturbation du sous-bois en intensité (disquage croisé, simple, inexistant) et en fréquence (trois, deux, une et zéro fois par an).

Les quatre peupleraies choisies correspondent aux principaux stades de développement d'une peupleraie artificielle et le changement d'entretien qui l'accompagne, de $\mathrm{P} 1$ récemment plantée, à $\mathrm{P} 4$, bientôt abattue. L'entretien de P3 est une condition particulière liée à des problèmes topographiques.

La fermeture du couvert et la quantité de lumière parvenant au sol est équivalente dans les peupleraies P2, P3 et P4 [20] contrastant ainsi avec la peupleraie P1, plus jeune. Tenant compte de la différence qui existe entre P1 et les trois autres peupleraies, nous insisterons ici sur les effets de l'entretien, principale cause de perturbation, et sur les réponses biotiques au niveau des communautés végétales.

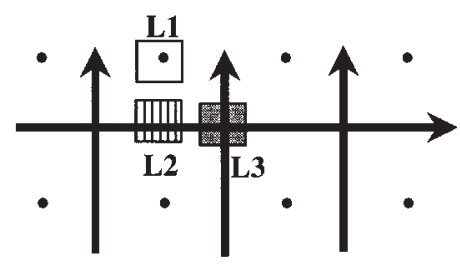

Entretien croisé

- P1 (trois fois par an)

- P2 (deux fois par an)

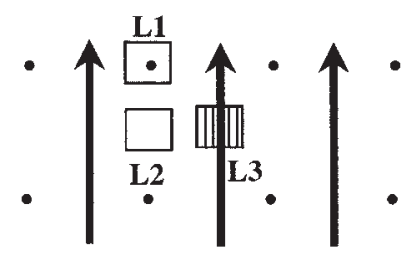

Entretien simple

- P3 (une fois par an)

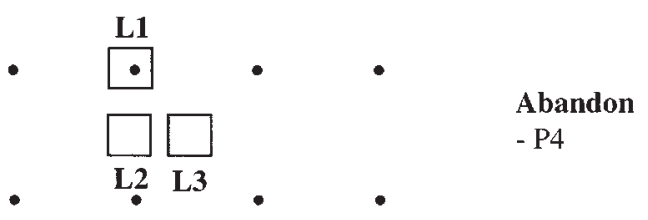

Figure 1. Modalités d'entretien des peupleraies étudiées et localisations L1, L2, L3 utilisées pour les emplacements des quadrats de prélèvement.

Perturbations locales liées à l'entretien : $\square$ absence de pertur-

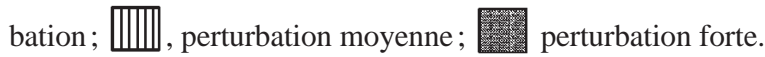

\section{3. Échantillonnage}

Afin de tenir compte de la variabilité de la perturbation au sein de chaque peupleraie, nous avons défini trois localisations de prélèvement (figure 1). Nous entendons par localisation, la position relative de chaque observation. Appelées L1, L2 et L3, elles sont respectivement situées : autour d'un peuplier, entre deux peupliers et au centre du carré formé par quatre arbres. Chaque localisation est définie à l'aide de quadrats de $1 \mathrm{~m}^{2}$ de surface (lorsque les quadrats sont placés autour des arbres (L1), la surface est calculée en tenant compte de l'aire de la base des troncs). Chacune des localisations est étudiée à l'aide de six répétitions. À partir d'une grille de repérage des différentes localisations possibles de chaque peupleraie, leur emplacement est défini par un traitement informatique utilisant un système de tirage au hasard (Méthode Monte-Carlo). À chaque manipulation, 
de nouveaux tirages sont effectués. Les quadrats utilisés sont éliminés des manipulations suivantes.

Nous avons réalisé un inventaire complet des espèces végétales (Cormophytes) des sous-bois. Cet inventaire résulte d'une prospection systématique mensuelle, de mars à septembre 1995, sur l'intégralité de la surface de chaque peupleraie. Les Bryophytes ont été nommées selon Husnot [16], les Ptéridophytes et les Angiospermes selon Tutin [39]. Les espèces recensées et déterminées ont été regroupées selon leur cycle de vie en annuelles, bisannuelles et vivaces. Les surfaces du peuplement forestier des quatre peupleraies sont différentes, les données sont corrigées selon Whittaker [42] : Richesse spécifique corrigée $=$ Nombre d'espèces $/ \log _{10}$ de l'aire d'étude.

Pour expliquer les variations de phytomasse et de richesse spécifique, nous avons retenu trois dates de prélèvement : mars (début de la période de végétation), juin (maximum de développement végétatif avant les premiers entretiens) et septembre (fin des principales phases de croissance). Les récoltes, opérées sur les six répétitions de chacune des localisations, ont été effectuées au cours des quinze premiers jours du mois concerné. Le prélèvement de la végétation est opéré par coupe manuelle au niveau du collet. À chaque prélèvement, nous mesurons la richesse et la composition spécifique par comptage et détermination de toutes les espèces présentes. Les espèces collectées sont placées dans une étuve à $105{ }^{\circ} \mathrm{C}$ et séchées jusqu'à poids constant. Après pesée, nous déterminons la phytomasse, regroupant biomasse et nécromasse (sur pied et détachée).

À l'aide des résultats obtenus, nous calculons la diversité $(H)$ à l'aide de l'indice de Shannon Wiener [34] : $-\Sigma p_{i} \log _{2} p_{i}$, avec $p_{i}$, rapport entre le poids de l'espèce $i$ et le poids total du relevé; ainsi que l'équitabilité $(J)$ selon [30] : $J=H / \log _{2}(n)$, avec $\log _{2}(n)$ diversité maximale pour un nombre $n$ d'espèces.

Lors de la récolte du mois de septembre, la peupleraie P1 a fait l'objet d'une opération de disquage en juin et en août, et se trouve à la veille d'un troisième entretien. La peupleraie P2 a été entretenue deux fois en juillet et en août. Quant à P3, elle a été entretenue au mois de juillet. D'une année à l'autre les dates des interventions peuvent varier en fonction des variabilités des conditions climatiques ou de la disponibilité du propriétaire, mais les fréquences (nombre d'opérations par an), inhérentes à l'état des arbres, sont identiques.

\subsection{Analyse des données}

Afin d'évaluer les différences observées dans les caractéristiques des communautés végétales, différence entre les peupleraies (P1 à P4) ou entre les points d'observation (localisations) d'une même peupleraie, nous avons utilisé un test de comparaison multiple des moyennes de Tukey [38]. Les données obtenues ont subi une transformation logarithmique afin de les normaliser.

Toutes les analyses ont été réalisées avec SYSTAT v. 8.

\section{RÉSULTATS}

\subsection{Richesse spécifique et composition des peupleraies étudiées}

L'inventaire global montre que les quatre peupleraies étudiées présentent des richesses spécifiques (valeurs transformées) nettement distinctes (tableau I). On observe que la richesse spécifique est d'autant plus élevée que le régime d'entretien est intense, de la peupleraie la moins entretenue $\mathrm{P} 4$ (38 espèces) à la plus entretenue $\mathrm{P} 1$ (146 espèces). Cette relation est identique pour les proportions des espèces annuelles et bisannuelles, mais inverse pour les vivaces.

La phytomasse est concentrée sur peu d'espèces (tableau II). Bien souvent, dans une peupleraie, la ou les espèces les plus développées représentent plus de $25 \%$ du poids total du relevé. Ce qui n'est pas le cas de la peupleraie $\mathrm{P} 1$, très entretenue. La diminution du régime d'entretien, de P1 à P4, facilite la dominance de certaines espèces. La peupleraie P1 (entretien croisé, fréquence 3 ) comporte des espèces de milieux ouverts, fortement perturbés (Cerastium glomeratum, Veronica persica). La peupleraie P2, moins entretenue (entretien croisé, fréquence 2), présente des espèces à durée de vie plus longue avec une forte présence d'espèces de graminées, le plus souvent stolonifères, comme Poa trivialis, Arrhenatherum elatius ou Agrostis stolonifera. Des espèces ligneuses comme Rubus caesius commencent à apparaître parmi les dominantes. Avec diminution ou arrêt des interventions comme c'est le cas dans P3 et P4,

Tableau I. Richesse spécifique obtenue (valeurs brutes et transformées) par inventaire complet de chaque peupleraie étudiée et pourcentage d'annuelles, de bisannuelles et de vivaces.

\begin{tabular}{lcccc}
\hline & $\mathrm{P} 1$ & $\mathrm{P} 2$ & $\mathrm{P} 3$ & $\mathrm{P} 4$ \\
\hline Richesse spécifique & & & & \\
Valeurs brutes & 146 & 96 & 67 & 38 \\
Valeurs transformées & 35,8 & 25,9 & 15,9 & 9,5 \\
Annuelles (\%) & 42,5 & 38,5 & 29,8 & 5,3 \\
Bisannuelles (\%) & 10,3 & 3,1 & 3 & 2,6 \\
Vivaces (\%) & 47,3 & 58,3 & 67,2 & 92 \\
\hline
\end{tabular}


Tableau II. Importance des espèces (\%) dans les quatre peupleraies étudiées au cours des différentes périodes de récolte : mars, juin et septembre 1995. Seules les espèces qui représentent plus de $5 \%$ du poids total de l'échantillon sont indiquées.

\begin{tabular}{|c|c|c|c|c|c|c|}
\hline \multicolumn{3}{|c|}{ Mars } & \multicolumn{2}{|l|}{ Juin } & \multicolumn{2}{|l|}{ Septembre } \\
\hline & Espèces & $\begin{array}{c}\text { Phyt. Rel. } \\
\text { (\%) }\end{array}$ & Espèces & $\begin{array}{c}\text { Phyt. Rel. } \\
\text { (\%) }\end{array}$ & Espèces & $\begin{array}{c}\text { Phyt. Rel. } \\
(\%)\end{array}$ \\
\hline \multirow[t]{7}{*}{ P1 } & Veronica persica & 19,3 & Poa trivialis & 14,3 & Conyza canadensis & 35,4 \\
\hline & Poa trivialis & 15,7 & Arrhenatherum elatius & 12 & Rubus caesius & 15,1 \\
\hline & Poа аппиа & 8,9 & Rubus caesius & 10,6 & Sonchus oleraceus & 11,7 \\
\hline & Cerastium glomeratum & 7,1 & Veronica persica & 8,8 & Sonchus asper & 7,5 \\
\hline & Alopecurus myosuroides & 6,9 & Galium aparine & 6,1 & Epilobium parviflorum & 6,3 \\
\hline & Rubus caesius & 5,1 & Cerastium glomeratum & 6 & Elymus repens & 5,9 \\
\hline & & & Valerianella locusta & 5,3 & & \\
\hline \multirow[t]{5}{*}{$\mathbf{P 2}$} & Poa trivialis & 27,1 & Arrhenatherum elatius & 29,9 & Rubus caesius & 42,4 \\
\hline & Arrhenatherum elatius & 24,4 & Rubus caesius & 18,7 & Arrhenatherum elatius & 20,8 \\
\hline & Arum italicum & 7,2 & Poa trivialis & 17,5 & Agrostis stolonifera & 13,7 \\
\hline & Galium aparine & 6,9 & Galium aparine & 7,1 & Hypericum perforatum & 8,4 \\
\hline & Agrostis stolonifera & 5,2 & & & & \\
\hline \multirow[t]{6}{*}{$\mathbf{P 3}$} & Urtica dioica & 38,5 & Urtica dioica & 32,2 & Rubus caesius & 45,2 \\
\hline & Poa rivialis & 19,9 & Rubus caesius & 26,4 & Urtica dioica & 21,9 \\
\hline & Rubus caesius & 19,9 & Poa trivialis & 7,9 & Phalaris arundinacea & 10,3 \\
\hline & Prunus domestica & 9,1 & Agrostis stolonifera & 7,7 & & \\
\hline & Ranunculus ficaria & 8,6 & Galium aparine & 5,4 & & \\
\hline & Phalaris arundinacea & 5,6 & & & & \\
\hline \multirow[t]{4}{*}{ P4 } & Vitis $x$ vinifera & 42,7 & Rubus caesius & 41 & Rubus caesius & 46,5 \\
\hline & Rubus caesius & 25,1 & Cornus sanguinea & 38,7 & Cornus sanguinea & 40,6 \\
\hline & Cornus sanguinea & 23,9 & Fraxinus angustifolia & 12,5 & Fraxinus angustifolia & 7,2 \\
\hline & Equisetum telmateia & 5,8 & Vitis $x$ vinifera & 6,5 & & \\
\hline
\end{tabular}

on voit apparaître des espèces dont la dominance est souvent élevée (> $40 \%$ ). Rubus caesius et Urtica dioica, pour la peupleraie $\mathrm{P} 3$, et des espèces sylvicoles pour $\mathrm{P} 4$ : Cornus sanguinea et Fraxinus angustifolia, ainsi qu'une liane Vitis $x$ vinifera.

Tableau III. Diversité et équitabilité (moyenne \pm intervalle de confiance à $95 \%$ ) des communautés végétales de sous-bois dans les quatre peupleraies étudiées au cours des différentes périodes de récolte.

\begin{tabular}{lcccc}
\hline & P1 & P2 & P3 & P4 \\
\hline Diversité (bits) & & & & \\
Mars & $2,9 \pm 0,2$ & $1,7 \pm 0,2$ & $1,3 \pm 0,2$ & $0,8 \pm 0,3$ \\
Juin & $3,1 \pm 0,2$ & $1,8 \pm 0,2$ & $1,3 \pm 0,1$ & $0,7 \pm 0,1$ \\
Septembre & $2,3 \pm 0,1$ & $1,4 \pm 0,1$ & $1 \pm 0,1$ & $0,7 \pm 0,1$ \\
Équitabilité & & & & \\
Mars & $0,7 \pm 0,05$ & $0,6 \pm 0,04$ & $0,5 \pm 0,01$ & $0,4 \pm 0,08$ \\
Juin & $0,7 \pm 0,06$ & $0,6 \pm 0,07$ & $0,4 \pm 0,03$ & $0,3 \pm 0,01$ \\
Septembre & $0,5 \pm 0,07$ & $0,4 \pm 0,07$ & $0,3 \pm 0,08$ & $0,3 \pm 0,07$ \\
\hline
\end{tabular}

Dans tous les cas, c'est en septembre que les dominances des espèces sont plus élevées. Les peupleraies les moins soumises au disquage montrent une diminution du nombre des espèces principales.

Ces observations se retrouvent dans les valeurs de diversité et d'équitabilité (tableau III) qui sont plus élevées pour les peupleraies subissant les plus forts entretiens. L'entretien diminue ainsi la dominance des espèces.

\subsection{Analyse du rôle des gradients de perturbations sur la richesse spécifique et la phytomasse des communautés de sous-bois \\ Comparaison inter-peupleraies}

La comparaison des différentes peupleraies entre elles permet de dire que celles-ci présentent des différences plus significatives pour la richesse spécifique que pour la phytomasse (tableau IV). 
Tableau IV. À partir des résultats obtenus au cours des trois périodes de prélèvement (mars, juin et septembre 1995), effet de l'intensité de la perturbation, liée à la peupleraie, sur les valeurs de richesse spécifique et de phytomasse. Moyenne des erreurs standard et probabilités associées au test de Tukey.

\begin{tabular}{lcc}
\hline & Richesse spécifique & Phytomasse \\
\hline P1P2 & $-0,21^{* * *}$ & $\mathrm{~ns}$ \\
P1P3 & $-0,39^{* * *}$ & $0,41^{* *}$ \\
P1P4 & $-0,67 * * *$ & $0,45^{* *}$ \\
P2P3 & $-0,18^{* *}$ & $\mathrm{~ns}$ \\
P2P4 & $0,46^{* * *}$ & $-0,33^{*}$ \\
P3P4 & $0,28^{* * *}$ & $\mathrm{~ns}$ \\
\hline
\end{tabular}

Note: $*, p<0.05 ; * *, p<0.01 ; * * *, p<0.001$.

La dynamique annuelle de toutes les peupleraies montre que les valeurs de richesse spécifique et de phytomasse sont élevées dès le mois de mars (figure 2). On observe les maxima en juin et on enregistre des diminutions en septembre. Pour les différentes dates d'observation, les valeurs obtenues dans les peupleraies font apparaître une augmentation de la richesse spécifique et, inversement une diminution de la phytomasse avec l'augmentation du niveau des perturbations quand on passe de la peupleraie la moins perturbée, P4, à la plus perturbée, P1. Mais les valeurs moyennes des peuple- raies les moins entretenues, $\mathrm{P} 3$ et $\mathrm{P} 4$, ne présentent que très peu de variation au cours des périodes étudiées.

Pour les valeurs de richesse spécifique, les différences enregistrées sont significativement différentes quelle que soit la période d'étude. Les phytomasses des peupleraies les moins entretenues P3 et P4, présentent une forte variabilité (intervalles de confiance larges)

Cette variabilité entraîne des différences non significatives entre les moyennes.

\section{Comparaison intra-peupleraie}

Concernant la richesse spécifique et la phytomasse, il n'existe que peu d'écarts significatifs entre les localisations d'une même peupleraie (figure 3). Cependant, certaines tendances apparaissent. Ainsi, dans les peupleraies entretenues, les richesses spécifiques augmentent avec l'intensité d'exposition à l'entretien, sauf dans la peupleraie la plus entretenue, P1, où celles ci diminuent en L3, localisation la plus perturbée. La phytomasse évolue de façon inverse.

Dans la peupleraie non perturbée, P4, la localisation L1, autour des arbres, possède des valeurs de phytomasse plus élevées, mais plus variables que les localisations 2 et 3 , entre les arbres. L'exemple illustré par le tableau $V$ indique que la localisation L1 présente une végétation plus riche en espèces ligneuses que les localisations L2 et L3.
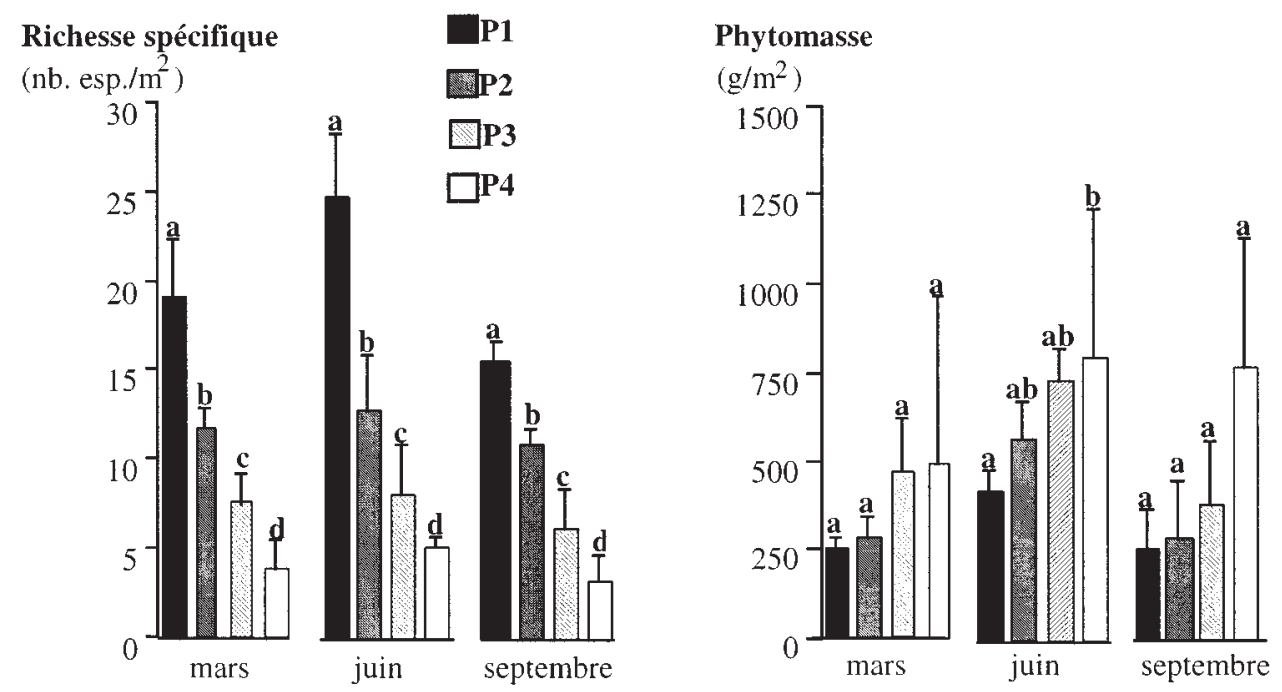

Figure 2. Dynamique annuelle des richesses spécifiques et des phytomasses pour les quatre peupleraies étudiées P1, P2, P3 et P4 (moyenne des six répétitions de chacune des localisations L1, L2 et L3 \pm Intervalle de confiance à $95 \%$ ). Les moyennes de la même période de récolte, qui ne sont pas suivies de la même lettre, sont significativement différentes selon un test de Tukey $(p<0,05)$. 
Richesse spécifique

(nb. esp. $/ \mathrm{m}^{2}$ )

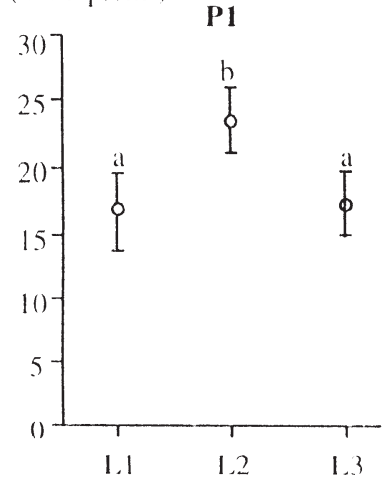

Phytomasse

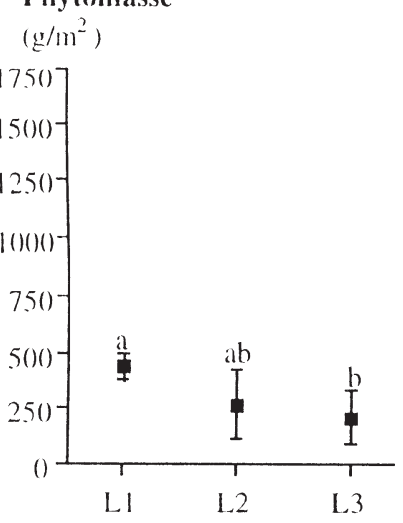

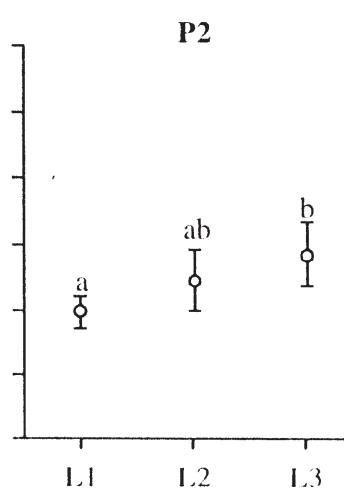
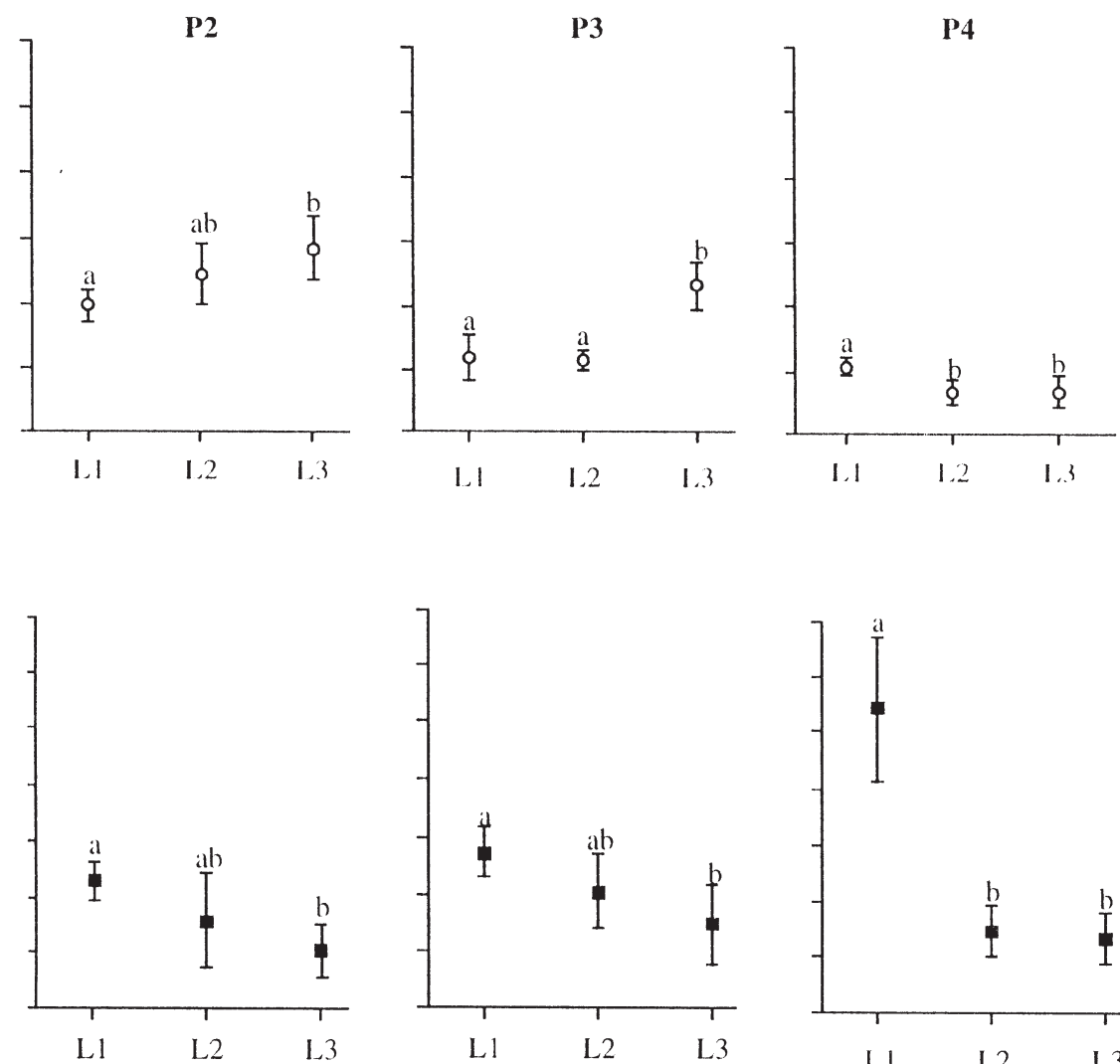

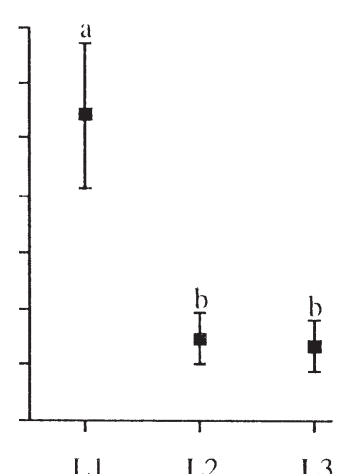

LI L2 L3

Figure 3. Richesse spécifique et phytomasse (moyenne des différentes dates de prélèvement \pm I. C. à $95 \%$ ) observées dans les quadrats localisés en L1, L2 et L3 pour les quatre peupleraies étudiées P1, P2, P3 et P4. Pour une même peupleraie, les moyennes, qui ne sont pas suivies de la même lettre, sont significativement différentes selon un test de Tukey $(p<0,05)$.

Tableau V. Liste des espèces rencontrées au mois de septembre, dans les localisations L1, L2 et L3 de la peupleraie P4. Chaque localisation est représentée par le relevé de ses six répliques.

\begin{tabular}{|c|c|c|c|}
\hline & L1 & $\mathrm{L} 2$ & L3 \\
\hline $\begin{array}{l}\text { Mousses } \\
\text { Brachythecium rutabulum }\end{array}$ & + & & \\
\hline $\begin{array}{l}\text { Herbacées } \\
\text { Brachypodium sylvaticum } \\
\text { Epilobium obscurum } \\
\text { Equisetum telmateia } \\
\text { Urtica dioica }\end{array}$ & $\begin{array}{l}+ \\
+ \\
+\end{array}$ & $\begin{array}{l}+ \\
+\end{array}$ & $\begin{array}{l}+ \\
+\end{array}$ \\
\hline $\begin{array}{l}\text { Ligneux } \\
\text { Cornus sanguinea } \\
\text { Fraxinus angustifolia subsp oxyphylla } \\
\text { Hedera helix } \\
\text { Rubus caesius }\end{array}$ & $\begin{array}{l}+ \\
+ \\
+ \\
+\end{array}$ & + & + \\
\hline
\end{tabular}

\section{DISCUSSION}

L'entretien des peupleraies par «disquage» représente une cause de perturbations, subies par les communautés végétales de sous-bois. Si l'on considère avant tout la perturbation comme élément de destruction [11], elle contribue bien souvent à l'augmentation de l'hétérogénéité environnementale [31] favorisant ainsi la diversité [5]. C'est ainsi que dans les peupleraies, la richesse spécifique atteint un maximum dans la localisation exposée à un entretien dont l'intensité de perturbation est moyenne. Ce cas est observé en (L2) de la peupleraie la plus perturbée $(\mathrm{P} 1)$, et se trouve conforme à l'hypothèse de la perturbation intermédiaire de Connell [7]. Dans les peupleraies les moins perturbées, P2 et P3, on observe une augmentation de la richesse spécifique en fonction de l'intensité d'exposition à l'entretien. En cas d'arrêt des entretiens, il y a une diminution de la richesse spécifique 
de la localisation située autour de l'arbre L1, à celles situées entre les arbres L2-L3. Les formations végétales ligneuses qui se développent dans ces deux localisations bloquent la dynamique des communautés de sous-bois tendant ainsi, par effet de compétition, à diminuer la richesse spécifique [17].

Cependant, si l'on se place à l'échelle de la peupleraie, on n'observe pas de situation intermédiaire : la richesse spécifique diminue en fonction de la perturbation de la peupleraie la plus perturbée P1 à la moins perturbée P4. Il semble que dans notre étude, nous ne soyons pas en présence de conditions extrêmes. Pour mieux étudier le phénomène, il faudrait prendre en compte des peupleraies dont les fréquences d'entretien sont supérieures. P1 est, de nos peupleraies, la plus perturbée, mais aussi la plus riche en espèces, par l'apparition d'espèces liées aux perturbations [12]. Ceci est favorisé par un milieu très ouvert présent uniquement en P1. Les peupleraies, par les caractéristiques de l'entretien, combinent la richesse spécifique de milieux perturbés (surtout en localisation L2 et L3) et de milieux stables (essentiellement en L1, jamais touchée par le disque d'entretien). Plus cette combinaison est favorisée par l'entretien, plus la richesse spécifique est élevée. Les milieux perturbés facilitent l'implantation d'espèces ubiquistes, essentiellement annuelles. Au contraire, les espaces protégés contribuent au développement d'espèces de milieux stables, la plupart vivaces [13]. L'hétérogénéité spatiale influe sur les valeurs de richesse spécifique [8]. Dans le cas étudié, c'est l'entretien qui créé cette hétérogénéité. En effet, celui-ci agit de manière différente selon le mode de disquage et l'emplacement étudié dans la peupleraie. Le travail du sol augmente ainsi les potentialités d'établissement des espèces, les capacités de germination des graines et la réduction de la concurrence des espèces déjà installées [18]. Mais, les différences de richesse spécifique sont plus marquées d'une peupleraie à une autre qu'au sein d'un même mode d'exploitation. La fréquence d'entretien, qui différencie les peupleraies, semble prévaloir sur son intensité. La réduction des passages des disques d'entretien instaure une certaine stabilité au sein de ces communautés de sous-bois et la richesse spécifique générale reste alors inchangée au cours de la période d'observation.

L'environnement très productif de plaine alluviale [22] présente ici deux avantages. D'une part, les perturbations observées sont à l'origine de fortes valeurs de richesses spécifiques, en accord avec les modèles de Connell [7] et de Huston [17]. D'autre part, les communautés observées réagissent vite à une perturbation [37]. En effet dès le mois de septembre, ces valeurs sont très vite récupérées. La forte humidité, liée à la proximité de la nappe, renforce ces phénomènes [26].
Cependant l'effet bénéfique du passage du disque d'entretien vis-à-vis de la richesse spécifique est un phénomène éphémère. Une étude récente [21] a montré que, l'observation de plantations très âgées (plus de 30 ans) et non entretenues depuis de plusieurs années, permet une augmentation de la richesse spécifique par l'apparition de nombreuses espèces de milieux plus stables, à durée de vie plus élevée.

La phytomasse suit des évolutions inverses de celles de la richesse spécifique. L'action mécanique du disque est facilement compréhensible. Aune et al. [4] font le même constat à propos de la diminution de biomasse en fonction de la fréquence de fauche. Dans la peupleraie la plus entretenue, P1, ni la fertilité du substrat, ni l'ouverture du milieu, qui normalement facilitent la croissance des espèces [5], ne permettent une forte accumulation de phytomasse. Ce constat est observable au sein des peupleraies entretenues. C'est ainsi que l'augmentation de l'intensité de perturbation, de L1 à L3, fait diminuer les valeurs de phytomasse. Comme le montrent Large et al. [22], la présence d'une hétérogénéité spatiale liée aux caractéristiques de la perturbation, ici l'entretien, contribue à la variabilité des valeurs de production. La peupleraie $\mathrm{P} 4$, abandonnée, présente de fortes valeurs de phytomasse en localisations L1, jamais entretenues. Les résultats obtenus sont dus à un fort développement de ligneux arbustifs ou de lianes ; ainsi il existe une forte variabilité entre les communautés des localisations L1 et celles de L2-L3.

$\mathrm{Au}$ cours du vieillissement de l'exploitation forestière, on a donc transformation de la nature du sous-bois par diminution puis arrêt des entretiens et fermeture progressive du couvert. Dans ce contexte fortement anthropisé, on peut suivre une succession végétale : d'un stade pionnier, riche en espèces peu dominantes et de courte durée de vie, puis prairial, dominé par des graminées le plus souvent stolonifères et enfin sylvatique, composé d'un étage de Fraxinus angustifolia et de Cornus sanguinea. Dans les conditions les plus stables, on observe des communautés dont le cortège floristique se rapproche fortement de celui relevé dans les formations «post-pionnières» de la ripisylve par Carbiener et al. [6]. L'influence de l'environnement est alors prépondérante par rapport à celui des interventions menées dans les peupleraies.

\section{CONCLUSIONS}

Depuis quelques années, on assiste à l'émergence d'une véritable théorie des perturbations [29], qui prend en compte la notion d'échelles emboîtées [3]. Un développement récent de cette théorie a été de reconnaître le rôle des hétérogénéités spatiales au sein de sites 
perturbés [28] comme entre sites perturbés [25]. Ce régime d'entretien des peupleraies est créateur d'hétérogénéités. Cette hétérogénéité est plus marquée entre peupleraies (dont les niveaux d'entretien sont différents) qu'au sein d'une même peupleraie (où les expositions à l'entretien sont différentes). Il en résulte, pour les communautés végétales des sous-bois, l'existence d'une dynamique en mosaïque principalement des richesses spécifiques et secondairement des phytomasses.

Bien que les peupleraies soient souvent considérées comme des facteurs de banalisation de la flore [33, 43], notre étude tend à montrer que les communautés de sous-bois sont riches en espèces et à composition qui varie avec les modalités de l'entretien. De nombreuses espèces sont souvent rudérales et/ou nitrophiles [9]. Mais ces caractères se retrouvent de façon naturelle en milieu alluvial. La peupleraie peut servir de refuge à de nombreuses espèces et constituer localement des banques de graines. Ces caractéristiques peuvent faire face à l'appauvrissement de plus en plus marqué des ripisylves. En effet, le milieu alluvial est, en raison de la diminution des crues et de nombreux aménagements, en voie d'uniformisation [19].

Remerciements : Je remercie le Pr. G. Durrieu et le Dr E. Tabacchi pour leur aide dans la rédaction de cet article ainsi que M. Y. Bournaud et la maison de retraite de Verdun-sur-Garonne, propriétaires des peupleraies étudiées, qui m'ont permis de mener à bien mes recherches.

\section{RÉFÉRENCES}

[1] Agard J.B.R., Hubbard R.H., Griffith J.K., The relation between productivity, disturbance and the biodiversity of carribean phytoplankton: applicability of Huston's dynamic equilibrium model, J. Exp. Mar. Biol. Ecol. 202 (1996) 1-17.

[2] Alard D., Bance J.F., Frileux P.N., Grassland vegetation as an indicator of the main agro-ecological factors in a rural landscape: consequences for biodiversity and wildlife conservation in central Normandy (France), J. Environ. Manage. 42 (1994) 91-109.

[3] Allen T.F.H., Wytelo E.P., A hierarchical model for the complexity of plant communities, J. Theor. Biol. 101 (1983) 529-540.

[4] Aune E.I., Kubicek F., Moen A., Oien D.I., Biomass studies in semi-natural ecosystems influenced by scything at the Solendet nature reserve, central Norway, Ekol. Bratislava 15 (1996) 307-320.

[5] Belsky A.J., Effects of grazing, competition, disturbance and fire on species composition and diversity in grassland communities, J. Veg. Sci. 3 (1992) 187-200.

[6] Carbiener R., Schnitzler A., Walter J.M., Problèmes de dynamique et de définition des stations en milieu alluvial, Coll. Phytos. (1985) 655-686.
[7] Connell J.H., Diversity in tropical rain forests and coral reefs, Science 199 (1978) 1302-1310.

[8] Cornell M.V., Lawton J.H., Species interactions, local et regional processes, et limits to the richness of ecological communities: a theoretical perspective, J. Anim. Ecol. 61(1992) $1-12$.

[9] Daudon M., Flore et végétation des Basses Vallées Angevines (Maine et Loire) : effets de divers modes d'utilisation du milieu. L.P.O., C.E.E., Ministère de l'Environnement, 1994, 42 p. + annexes.

[10] Décamps H., Fortuné M., Gazelle F., Pautou G., Historical influence of man on the riparian dynamics of a fluvial landscape, Landscape Ecol. 1 (1988) 163-173.

[11] del Moral R., Titus J.H., Cook A.M., Early primary succession on Mount St Helens, Washington, USA, J. Veg. Sci. 6 (1995) 107-120.

[12] Denslow J.S., Tropical treefall gaps and tree species diversity, Annu. Rev. Ecol. Syst. 18 (1987) 431-451.

[13] Grime J.P., Plant strategies et vegetation processes, University of Sheffield, 1979.

[14] Halpern C.B., Spies T.A., Plant species diversity in natural and managed forests of the Pacific nothrwest, Ecol. Appl. 5 (1995) 913-934.

[15] Heywood V.H., Watson R.T., Global biodiversity assesment, U.N.E.P. Cambridge University Press, Cambridge, 1995, $1140 \mathrm{p}$.

[16] Husnot T., Muscologica Gallica. Descriptions et figures des mousses de France et des contrées voisines, Amsterdam, 1967, 458 p.

[17] Huston M., A general hypothesis of species diversity, Am. Nat. 113 (1979) 81-101.

[18] Kocher E., Stubbendieck J., Broadcasting grass seed to revegetate sandy soils, J. Range. Manage. 39 (1986) 555-557.

[19] Laquerbe M., Dynamique du paysage riverain des bords de la Garonne. Effets anthropiques, Bull. Soc. Hist. Nat. Toul. 133 (1997) 7-13.

[20] Laquerbe M., Dynamique des communautés végétales dans les sous-bois des peupleraies : effets des perturbations liées à l'entretien. Thèse Université Paul Sabatier, Toulouse, 1998, $193 \mathrm{p}$.

[21] Laquerbe M., Communautés de sous-bois des peupleraies artificielles : relation entre phytomasse, richesse spécifique et perturbations, Ann. For. Sci. 56 (1999) 607-614.

[22] Large A.R.G., Wade P.M., Pautou G., Amoros C., Producteurs et productions primaires, in: Amoros C., Petts G. (Eds.), Hydrosystèmes fluviaux, Masson, Paris, 1993, pp. 107-124.

[23] Lefeuvre J.C., Commentaires, Natures - Sciences Sociétés 3 (1995) 144-148.

[24] Malanson G.P., Riparian Landscape, Cambrige University Press, Cambridge, 1993, 296 p.

[25] Milchunas D.G., Lauenroth W.K., Chapman P.L., Kazempour M.K., Community attributes along a perturbation gradient in a short grass steppe, J. Veg. Sci. 1 (1990) 375-384. 
[26] Milchunas D.G., Lauenroth W.K., Quantitative effects of grazing on vegetation and soils over a global range of environments, Ecol. Monogr. 63 (1993) 323-326.

[27] Pearson P., Écologie forestière : la forêt, son climat, son sol, ses arbres, sa faune, Gauthier-Villars, Paris, 1974, $382 \mathrm{p}$.

[28] Peterson C.J., Pickett S.T.A., Forest reorganization: a case study in an old-growth forest catastrophic blowdown, Ecology 76 (1995) 763-774.

[29] Pickett S.T.A., Kolasa J., Aramesto J.J., Collins S.L., The ecological concept of disturbance and its expression at various hierarchical levels, Oikos 54 (1989) 129-136.

[30] Pielou E.C., Ecological Diversity, Wiley-Interscience, New-York, 1975.

[31] Poff N.L., Ward J.W., Physical habitat template of lotic systems: recovery in the context of historical pettern of spatiotemporal heterogeneity, Environ. Manage. 14 (1990) 629-645.

[32] Ricklefs R.E., Schulter D., Species diversity in ecological communities. Historical et geographical perspective, The University of Chicago Press, Chicago, London, 1993, 414 p.

[33] Schnitzler A., Conservation of biodiversity in alluvial hardwood forests of the temperate zone. The example of the Rhine valley, For. Ecol. Manage. 68 (1994) 385-398.

[34] Shannon C.E., A mathematical theory of communication, Bell Syst. Tech. J. 27 (1948) 379-423, 623-656.
[35] Sousa W.P., The role of disturbance in natural communities, Annu. Rev. Ecol. Syst. 15 (1984) 353-391.

[36] Tilman D., Ressource competition and community structure, Princeton University Press, Princeton, N.J., 1982, $276 \mathrm{p}$.

[37] Tilman D., Downing, J.A., Biodiversity et stability in grasslands, Nature 367 (1994) 363-365.

[38] Tukey J.W., Exploratory Data Analysis, AddisonWasley, Reading, PM, 1987.

[39] Tutin T.G., Heywood V.H., Burges N.A., Moore D.M., Valentine D.H., Walters S.M., Webb D.A., Flora europaea, Cambridge University Press, Cambridge, 5 volumes, 1964-1980.

[40] Ward J.V., Riverine landscapes: biodiversity patterns, disturbance regimes, and aquatic conservation, Biol. Conserv. 83 (1998) 269-278.

[41] White P.S., Pickett S.T.A., Natural disturbance et patch dynamics: an introduction, in: Pickett S.T.A., White P.S (Eds.), The ecology of natural disturbance et patch dynamics, Academic Press, Orleto, 1985, pp 3-13.

[42] Whittaker R.H., Evolution and measurement of species diversity, Taxon 21 (1972) 213-251.

[43] Yon D., Évolution des forêts alluviales en Europe, facteurs de destruction et éléments stratégiques de conservation, Coll. Phytos. Strasbourg 9 (1984) 1-18.

To access this journal online: www.edpsciences.org 